\title{
Distribución de la infraestructura verde y su capacidad de regulación térmica en Bogotá, Colombia
}

\author{
Distribution of the green infrastructure \\ and its thermal regulation capacity in Bogotá, Colombia
}

\author{
Kristian Rubiano ${ }^{10}$
}

Rubiano, K. (2019). Distribución de la infraestructura verde y su capacidad de regulación térmica en Bogotá, Colombia. Colombia Forestal, 22(2), 83-100.

Recepción: 26 de diciembre de 2018

Aprobación: 15 de abril de 2019

\section{Resumen}

Las ciudades presentan características ambientales particulares que dan lugar a fenómenos como las islas de calor. Aunque las coberturas vegetales son fundamentales en la mitigación de estas condiciones adversas, se ha observado que su distribución no es equitativa. Analizar esta distribución y la oferta de servicios ecosistémicos es importante para la planificación de las ciudades. Por tal razón, se analizó la distribución de la capacidad de regulación térmica de las coberturas vegetales públicas de la ciudad de Bogotá, usando la cobertura arbórea y el verdor como variables proxy, mediante análisis geoestadístico y metodologías propias de los sistemas de información geográfica y la teledetección. El verdor de los parques aumentó con su tamaño y estrato socioeconómico, la cobertura arbórea incrementó en los estratos más altos. Se identificaron coldspots y hotspots de estas variables y del potencial de regulación térmica en los sectores con estratos más bajos y más altos respectivamente.

Palabras clave: cobertura arbórea, índice de vegetación de diferencia normalizada, inequidad ambiental, isla de calor, parques urbanos, servicios ecosistémicos urbanos.

\begin{abstract}
Cities show particular environmental characteristics that give rise to phenomena such as heat islands. Although vegetation covers are key in mitigating these adverse conditions, their distribution has been found to be inequitable. Analyzing this distribution and the supply of ecosystem services is important for the city planning . For this reason, the distribution of the thermal regulation capacity of the public vegetation covers of Bogotá city was analyzed, using tree coverage and greenness as proxy variables, through geostatistical analysis, Geographic Information Systems and remote sensing. There was an increase in the average greenness of the parks according to their size and the socioeconomic stratum where they are located, the tree coverage increased in the highest strata. Coldspots and hotspots of these variables and the thermal regulation potential were identified in the areas with the lowest and highest strata, respectively.
\end{abstract}

Keywords: tree canopy cover, normalized difference vegetation index, environmental inequity, urban heat island, urban parks, urban ecosystem services.

Jardín Botánico de Bogotá José Celestino Mutis. Bogotá, Colombia. krubiano@jbb.gov.co, kristian.rubianoc@gmail.com. 


\section{INTRODUCCIÓN}

En los últimos siglos, como consecuencia del desarrollo económico y el crecimiento poblacional, se han presentado cambios significativos en el uso y cobertura de la tierra. El ejemplo más drástico de este proceso es la urbanización, la cual consiste en transformar y reemplazar los ecosistemas naturales por elementos artificiales como edificaciones y vías (Foley et al., 2005; Ellis y Ramankutty, 2008). Estas transformaciones, sumadas a las altas densidades poblacionales, generan unas condiciones ambientales particulares que afectan la calidad de vida de los habitantes urbanos (Patz, Campbell-Lendrum, Holloway y Foley, 2005; Basara, Basara, Illston y Crawford, 2010). Por esto, son fundamentales los servicios ecosistémicos que prestan las coberturas vegetales, al regular y mantener unas condiciones de vida sostenibles (Bolund y Hunhammar, 1999; Gómez-Baggethun et al., 2013; Lovell y Taylor, 2013).

Una de las características ambientales más contrastantes de las ciudades respecto a las áreas rurales aledañas es el aumento de la temperatura (Zhao et al., 2006; Peng et al., 2012; Livesley, McPhearson y Calfapietra, 2016). Este fenómeno conocido como isla de calor es producto principalmente de las características de los materiales empleados para la construcción de las ciudades, los cuales no permiten la evapotranspiración y absorben y retienen mayores cantidades de calor que la vegetación y otras coberturas naturales (Grimmond, 2007; Rizwan, Dennis y Liu, 2008). A su vez, la configuración espacial de las ciudades y la contaminación generada por las actividades diarias, incrementan el consumo de energía invertida para el enfriamiento de edificaciones, la demanda de combustibles fósiles y la emisión de gases de efecto invernadero (Santamouris, Cartalis, Synnefa y Kolokotsa, 2015). Por todo esto, la regulación de la temperatura es uno de los servicios ecosistémicos más relevantes que proveen las coberturas vegetales urbanas (McPhearson, 2011). A través de la evapotranspiración, el agua de las plantas absorbe calor y reduce la temperatura del aire, a la vez que se eleva la humedad relativa (Jim y Chen, 2009; Bowler, Buyung-Ali, Knight y Pullin, 2010). La provisión de sombra también regula la temperatura del aire y la temperatura superficial al reflejar la radiación solar y evitar que otras superficies absorban calor (Bolund y Hunhammar, 1999; Shashua-Bar y Hoffman, 2000; Hardin y Jensen, 2007; Bowler et al., 2010; Gómez-Baggethun et al., 2013).

Aunque la importancia del acceso a la vegetación urbana y sus servicios ecosistémicos es ampliamente reconocida, hay pruebas que demuestran que su distribución no es equitativa en muchas ciudades del mundo (Kabisch y Haase, 2014; Schwarz et al., 2015; Nesbitt y Meitner, 2016). Factores socioeconómicos como los ingresos monetarios, características étnicas y raciales, género, entre otros, han sido identificados como limitantes de la disponibilidad de coberturas vegetales urbanas tanto en cantidad como en calidad. Esto tiene implicaciones en la oferta de servicios ecosistémicos, la cual se ve disminuida en los grupos socioeconómicos menos favorecidos (Mitchell y Popham, 2008; Ernstson, 2013; Wolch, Byme y Newell, 2014). En Latinoamérica, en donde el 80 $\%$ de la población vive en ciudades y se presenta una marcada inequidad social (United Nations, 2018), se ha identificado que los residentes con mejores ingresos económicos tienen mayor acceso a espacios verdes y diversidad de especies arbóreas en ciudades como Santiago de Chile (De la Maza, Hernández, Brown, Rodríguez y Escobedo, 2002; Escobedo, Palmas-Perez, Dobbs, Gezan y Hernández, 2016) y Río de Janeiro (Pedlowski, Da Silva, Adell y Heynen, 2002). En el caso de Bogotá, se identificó menor abundancia de arbolado (Brown, 2012) y una reducción de sus parámetros estructurales en las zonas más pobres de la ciudad (Escobedo, Clerici, Staudhammer y Corzo, 2015). Esta problemática constituye una de las principales preocupaciones para la gestión urbana, en busca del bienestar generalizado y calidad de vida (Ernstson, 2013; Wolch et al., 2014). 
Si bien la atención prestada a los ecosistemas urbanos es baja si se compara con la recibida por bosques y otros ecosistemas naturales (Gómez-Baggethun y Barton, 2013), el aumento en el número de publicaciones relacionadas con el mapeo de los servicios ecosistémicos indica que es un campo de investigación emergente (Ochoa y Urbina-Cardona, 2017). Su contribución como herramienta de gestión y planeación del territorio, resalta su importancia en las áreas urbanas (Egoh et al., 2008; Maes et al., 2012). Diferentes metodologías y aproximaciones se han empleado para el estudio de la distribución y el mapeo de los servicios ecosistémicos (Egoh, Drakou, Dunbar, Maes y Willemen, 2012; Maes et al., 2012). Una de las más empleadas, y que busca lidiar con la falta de información primaria, es el uso de estimaciones basadas en el tipo de cobertura (Burkhard, Kroll, Müller y Windhorst, 2009; Burkhard, Kroll, Nedkov y Müller, 2012; Schneiders, Van Daele, Van Laduyt y Van Reeth, 2012). No obstante, estas estimaciones han presentado ajustes bajos con información primaria, por lo que son recomendadas para determinar patrones de distribución a escala gruesa (Eigenbrod et al., 2010). A escalas locales como las ciudades, los datos estructurales medidos en cada árbol a través de censos forestales, son de gran valor para el estudio de los servicios ecosistémicos, no obstante, pocas ciudades cuentan con inventarios forestales de este tipo debido a sus altos costos (Nowak et al., 2008).

Pese a su relevancia, el mapeo de la regulación térmica en las ciudades ha sido poco caracterizada debido a que generalmente se relaciona con el almacenamiento y captura de carbono. Sin embargo, los ecosistemas ofrecen este servicio a diferentes niveles: en las ciudades a través de la provisión de sombra y la evapotranspiración vegetal, y a escalas espaciales mayores a través del almacenamiento y captura de carbono, contribuyendo a la regulación climática global (Maes et al., 2017). Esto implica la intervención de diferentes funciones ecosistémicas y el uso de distintos indicadores para su estudio (Egoh et al., 2012). Como antecedente del mapeo de la capacidad de regulación de temperatura urbana, se encuentran los trabajos presentados por Zulian et al. (2017) y Cortinovis y Geneletti (2018), en los que a través de una metodología basada en literatura se identificó la provisión de sombra y la evapotranspiración como las funciones principales asociadas al servicio ecosistémico; y, posteriormente, mediante el uso de indicadores se generó un mapa representando la capacidad de regulación térmica en la ciudad de Trento, Italia. De manera similar, Zardo, Geneletti, Pérez-Soba y Van Eupen (2017) desarrollaron una metodología para evaluar el potencial de regulación térmica de la infraestructura verde, identificando nuevamente la provisión de sombra y la evapotranspiración como funciones principales, y luego la aplicaron en la ciudad de Ámsterdam.

El objetivo de esta investigación fue analizar la distribución espacial de la infraestructura verde pública de Bogotá y su capacidad de regulación térmica, en relación con la estratificación socioeconómica, esto con el fin de proveer información que contribuya al mejoramiento de la provisión de este y otros servicios ecosistémicos. De acuerdo con los antecedentes, se espera identificar diferencias en la distribución de la cobertura arbórea, el verdor de los parques públicos y por consiguiente del potencial de regulación de temperatura en las áreas de la ciudad con menor estrato socioeconómico.

\section{MATERIALES Y MÉTODOS}

\section{Área de estudio}

Bogotá es la capital y ciudad más grande de Colombia. Se localiza en el centro del país sobre la cordillera oriental, a una altitud promedio de 2630m (figura 1). Cuenta con una población de alrededor de 8 millones de habitantes (Cristancho y Triana, 2018), y una superficie urbana de aproximadamente $380 \mathrm{~km}^{2}$. Durante los últimos años, factores como la contaminación y la urbanización 
han hecho más cálida la ciudad y acentuado la presencia del efecto isla de calor en las zonas más internas, las cuales superan en aproximadamente $3{ }^{\circ} \mathrm{C}$ la temperatura de las áreas periféricas (Ángel, Ramírez y Domínguez, 2010).

La división política y administrativa de la ciudad comprende 19 localidades que, a su vez, se subdividen en 112 UPZ (Unidades de Planeamiento Zonal), que corresponden a áreas con usos del suelo y desarrollos urbanísticos similares (Secretaría Distrital de Planeación, 2009). La división socioeconómica se representa a través de los estratos socioeconómicos, los cuales fueron establecidos por el gobierno distrital como una aproximación para capturar las diferencias socioeconómicas entre zonas de la ciudad y como una herramienta para direccionar efectivamente el gasto público hacía los ciudadanos menos favorecidos (Hernández, 2016). Existen seis estratos socioeconómicos, de los cuales el uno corresponde a las zonas más pobres de la ciudad, con carencias en infraestructura y servicios públicos, mientras que el seis representa las zonas de la ciudad donde se concentran los mayores ingresos monetarios y una gran oferta de infraestructura y servicios (Brown, 2012; Escobedo et al., 2015).

Con respecto a la infraestructura verde, la ciudad cuenta con la denominada Estructura Ecológica Principal (EEP), que agrupa los elementos fundamentales para la conservación de la
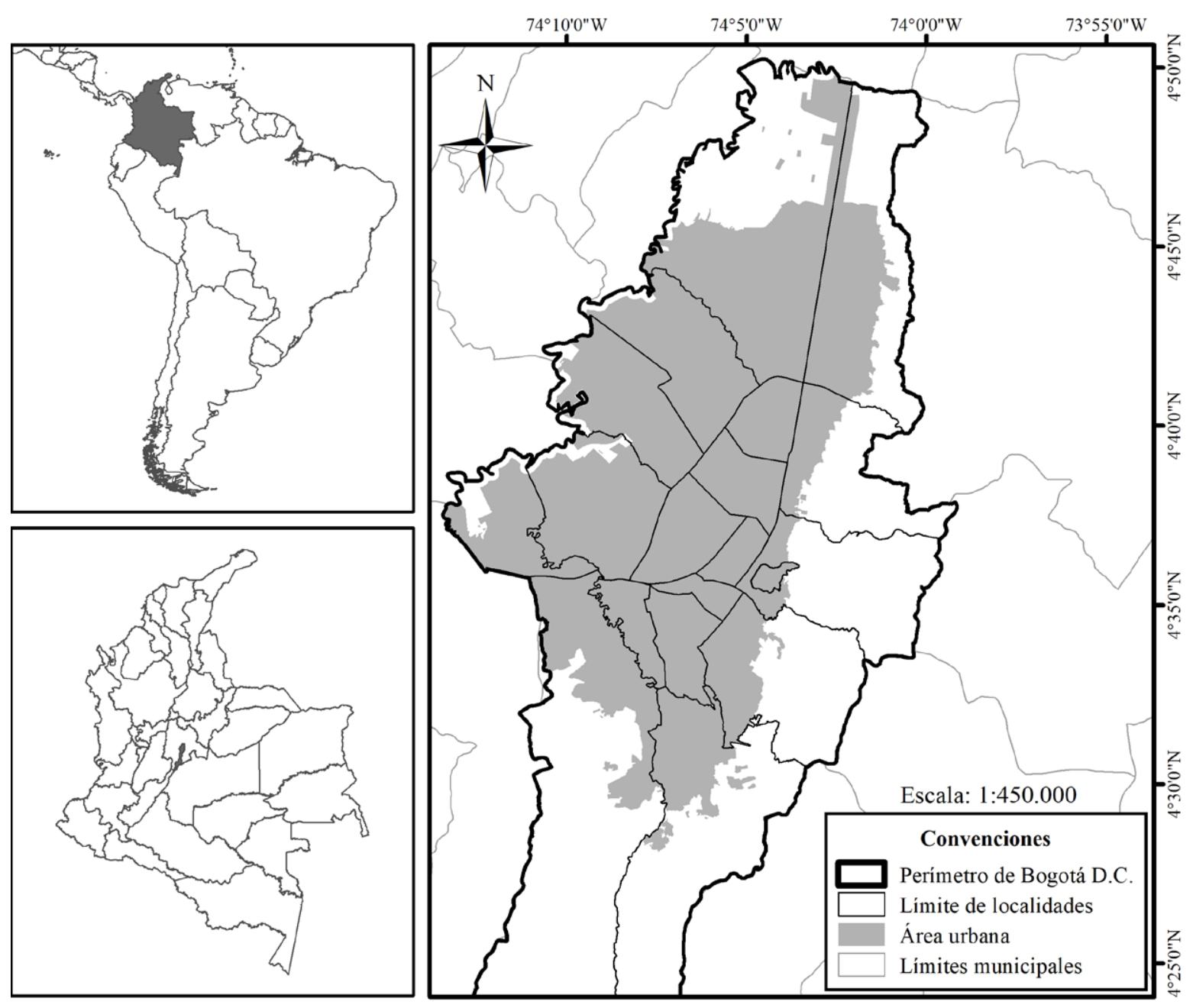

Figura 1. Localización del área de estudio. 
biodiversidad y los procesos ecológicos esenciales para el desarrollo sostenible (Isaacs y Jaimes, 2014). Dentro de los componentes de la EEP están los parques urbanos, espacios verdes de uso público que sirven como reguladores del equilibrio ambiental y brindan espacios de recreación y aprovechamiento para los ciudadanos. Los parques urbanos se clasifican en cuatro categorías de acuerdo con su tamaño y área de influencia: parques metropolitanos (> $10 \mathrm{ha}$ ), parques zonales (1 a $10 \mathrm{ha})$, parques vecinales $\left(1000 \mathrm{~m}^{2}\right.$ a $\left.1 \mathrm{ha}\right) \mathrm{y}$ parques de bolsillo $\left(<1000 \mathrm{~m}^{2}\right)$ (Secretaría Distrital de Planeación, 2009). El arbolado público de la ciudad se distribuye tanto en parques como en los diferentes tipos de espacio público tales como separadores, andenes, entre otros. Algunas de las especies más abundantes son el sauco (Sambucus nigra L.), el jazmín del Cabo (Pittosporum undulatum Vent.) y la acacia japonesa (Acacia melanoxylum R. Br.) (Mahecha et al., 2010).

\section{Estimación de la cobertura arbórea}

Para estimar la cobertura arbórea se obtuvo información del Sistema de Información para la Gestión del Arbolado Urbano de Bogotá (Sigau) (tabla 1), el cual consiste en un inventario del arbolado público de la ciudad. La base de datos incluye datos morfométricos de aproximadamente 1300000 individuos con altura igual o superior a 30 cm, y su localización en coordenadas geográficas (Dirpen, 2006). La cobertura arbórea se estimó con respecto a todos los individuos, modelando la proyección de la copa al suelo, asumiendo que el área de la copa corresponde a una circunferencia cuyo diámetro es equivalente al diámetro ecuatorial del individuo (Rodríguez-Laguna, Meza-Rangel, Vargas-Hernández y Jiménez-Pérez, 2009). Esta variable hace parte del Sigau y fue medida en terreno como la mayor longitud de la proyección ortogonal de la copa (Dirpen, 2006).

\section{Cálculo del índice de vegetación de diferencia normalizada (NDVI)}

Los índices de vegetación (IV) se fundamentan en las características físico-químicas de la vegetación que determinan su respuesta a la interacción con las diferentes longitudes de onda de la radiación electromagnética. La vegetación es altamente reflectiva en la región del espectro electromagnético correspondiente al infrarrojo cercano y a su vez presenta una baja reflectividad en la región del rojo. Por tal motivo, muchos de los IV usan las bandas del rojo y el infrarrojo cercano de las imágenes satelitales con el fin de acentuar las

Tabla 1. Fuentes de la información empleada para los análisis.

\begin{tabular}{|c|c|c|}
\hline Tipo de información & Descripción & Fuente \\
\hline $\begin{array}{l}\text { Capa del arbolado públi- } \\
\text { co de Bogotá }\end{array}$ & $\begin{array}{l}\text { Capa de puntos con atribu- } \\
\text { tos del arbolado de la ciudad }\end{array}$ & $\begin{array}{l}\text { Sistema de Información para la Gestión del Arbolado Urbano de } \\
\text { Bogotá (SIGAU) http://www.jbb.gov.co/index.php/sigau }\end{array}$ \\
\hline Imagen multiespectral & $\begin{array}{l}\text { Imagen Landsat } 8 \text { con } \\
\text { identificador del producto } \\
\text { LC } 80080572015052 \text { LGN01 }\end{array}$ & U.S. Geological Survey (USGS) https://earthexplorer.usgs.gov/ \\
\hline $\begin{array}{l}\text { Capa de unidades de } \\
\text { planeamiento zonal (UPZ) }\end{array}$ & $\begin{array}{l}\text { Subdivisión urbana de } \\
\text { Bogotá }\end{array}$ & \\
\hline Estratos socioeconómicos & $\begin{array}{l}\text { Estrato socioeconómico a } \\
\text { nivel de lote }\end{array}$ & $\begin{array}{l}\text { Infraestructura de Datos Espaciales para el Distrito Capital (IDECA) } \\
\text { https://www.ideca.gov.co }\end{array}$ \\
\hline Capa de lotes & Lotes de la ciudad & \\
\hline Capa de localidades & División urbana de Bogotá & \\
\hline Capa de parques urbanos & $\begin{array}{l}\text { Capa de parques urbanos } \\
\text { con atributo de clasificación }\end{array}$ & $\begin{array}{l}\text { Secretaría Distrital de Ambiente http://www.secretariadeambiente. } \\
\text { gov.co/visorgeo } \\
\text { Portal de mapas de Bogotá http://mapas.bogota.gov.co/portalmapas/ }\end{array}$ \\
\hline
\end{tabular}


propiedades espectrales de la vegetación y atenuar las características de las demás coberturas (Chuvieco, 2016). El NDVI, uno de los IV más comunes, tiene numerosas aplicaciones en estudios ecológicos (Kerr y Otrovsky, 2003; Glenn, Huete, Nagler y Nelson, 2008), incluyendo el análisis y cuantificación de servicios ecosistémicos (Ayanu, Conrad, Nauss, Wengmann y Koellner, 2012). Múltiples autores han empleado este índice como una medida de verdor en zonas urbanas como resultado de la cantidad de vegetación o coberturas permeables presentes en un área (Zhou, Troy, Grove y Jenkins, 2009; Leslie, Sugiyama, lerodiaconou y Kremer, 2010). Adicionalmente, diferentes estudios han encontrado una relación inversa entre la temperatura de la superficie y el NDVI (Weng et al., 2004; Weng, 2009; Huang y Ye, 2015).

Para calcular el NDVI, se utilizó una imagen multiespectral obtenida por el sensor OLI a bordo de la plataforma Landsat 8 (tabla 1). La cobertura de nubes alcanzó el $25 \%$, pero no hubo incidencia sobre el área de interés. La imagen se adquirió preprocesada, tanto geométrica (Storey, Choate y Lee, 2014) como radiométricamente (Chander, Markham y Helder, 2009) y transformada a unidades de reflectancia de la superficie por el Servicio Geológico de los Estados Unidos, mediante la aplicación del algoritmo LaSRC (Landsat Surface Reflectance Code) (United States Geological Survey, 2017). De esa forma, se obtuvo como resultado una estimación de la reflectancia espectral de la superficie que puede ser aplicada para estudios de vegetación y el cálculo más preciso de índices espectrales (Chuvieco, 2016). Sobre esta imagen se calculó el NDVI así (Rouse, Haas, Schell, Deering y Harlan, 1974):

Donde, IRC corresponde a los valores de reflectancia de la banda del infrarrojo cercano (OLI5) y ROJO a los valores de reflectancia de la banda del rojo (OLI4). El intervalo del NDVI va de -1 a 1 , pero la vegetación generalmente registra valores por encima de 0.1 , donde los valores más altos están asociados con mayor densidad de coberturas vegetales saludables (Jensen, 2000). Por tal motivo, se reclasificó para obtener valores entre 0 y 1 , intervalo en el que se hace énfasis en el verdor.

\section{Análisis espacial}

Se usaron las capas de cobertura arbórea y NDVI previamente generadas, y otras capas del mapa de referencia para Bogotá en la versión correspondiente al mes de junio de 2017, el visor geográfico de la Secretaría Distrital de Ambiente y el portal de mapas de Bogotá (tabla 1). Los procesos y herramientas necesarios para los análisis se ejecutaron en el software QGIS 2.18 (QGIS Development Team, 2011). Para determinar la distribución de la cobertura arbórea en las zonas de la ciudad con diferente estrato socioeconómico, se realizó un análisis desde el ámbito de las UPZ. Para ello, se generaron capas de cobertura arbórea y estrato socioeconómico por UPZ que posteriormente se cruzaron para obtener el porcentaje del área total de cada estrato socioeconómico representada por cobertura arbórea (figura 2). En el caso del NDVI se obtuvo valores medios para cada UPZ y para cada parque público a partir de los valores registrados en cada píxel. Como consecuencia de la variación en la forma y tamaño de los parques, y la resolución espacial de la imagen satelital $\left(900 \mathrm{~m}^{2}\right)$, los polígonos no se distribuyeron de manera uniforme sobre los píxeles. Para lidiar con este problema, el NDVI se remuestreó con un tamaño de píxel de 1 $\mathrm{m}^{2}$ antes de la extracción de las estadísticas zonales, ponderando así cada píxel de tamaño original por el área intersectada por cada uno de los polígonos de los parques (Wilson et al., 2016).

\section{Mapeo de la capacidad de regulación térmica}

Inicialmente, se identificaron la provisión de sombra y la evapotranspiración como las funciones principales que influencian la capacidad de la infraestructura verde para la regulación térmica (Zardo et al., 2017; Zulian et al., 2017; Cortinovis y Geneletti, 2018). Se usó el porcentaje de cobertura 


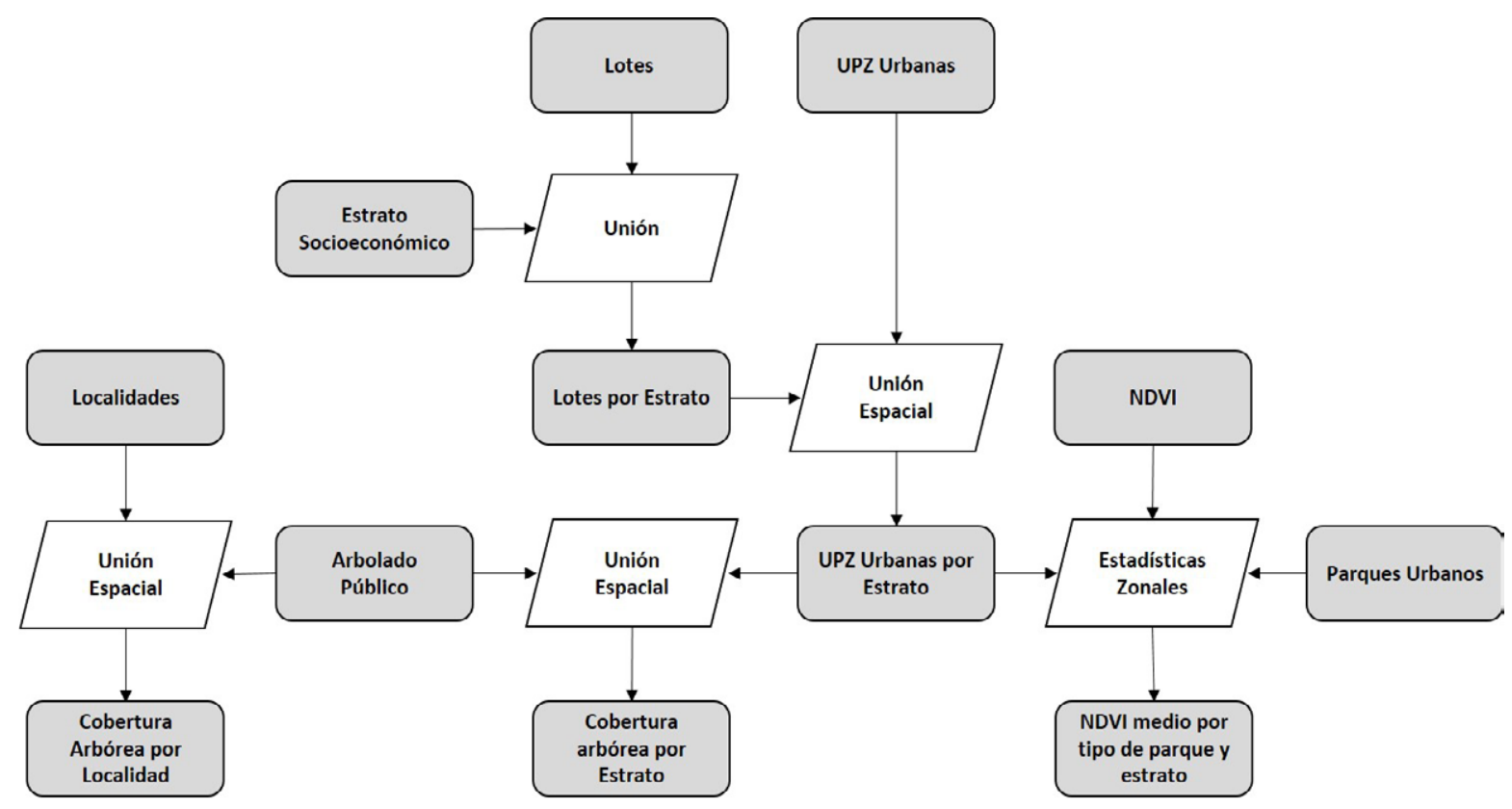

Figura 2. Flujo de trabajo del análisis espacial.

arbórea como proxy de la cantidad de sombra ofrecida por el arbolado urbano (Shashua-Bar y Hoffman, 2000), la cual ha sido ampliamente reconocida como uno de los mecanismos de regulación de temperatura en las ciudades (Bolund y Hunhammar, 1999; Hardin y Jensen, 2007; Bowler et al., 2010; Gómez-Baggethun et al., 2013). La media del NDVI de los parques públicos de cada UPZ, transformado a valores entre 0 y 100, se usó como proxy del verdor o abundancia de coberturas vegetales o superficies con capacidad de evapotranspirar (Zhou et al., 2009; Leslie et al., 2010), teniendo en cuenta que el aumento en el NDVI se ha relacionado con la disminución de las temperaturas urbanas (Weng et al., 2004; Weng, 2009; Huang y Ye, 2015).

Posteriormente, se evaluó cada una por separado y finalmente se combinaron para generar un mapa coroplético que representa el potencial de regulación térmica de la infraestructura verde pública de Bogotá con respecto a las UPZ. La combinación de estás dos variables para determinar dicho potencial, corresponde a su importancia para la regulación climática urbana y lo señalado en las metodologías propuestas por Zardo et al. (2017), Zulian et al. (2017) y Cortinovis y Geneletti (2018). Respecto a la contribución relativa de las variables a la capacidad de regulación térmica, se ha identificado que varía en función del tamaño del área analizada cuando se toman parques y elementos específicos de la infraestructura verde como unidad de análisis, pero no hay suficiente información en la literatura sobre cómo combinar su contribución (Zardo et al., 2017). Por lo anterior, y teniendo en cuenta que no se consideró el área de los parques o las UPZ como un factor de análisis, se optó por seleccionar una contribución balanceada, asignando los mismos pesos a las dos variables. De esa manera, cada UPZ obtuvo un puntaje entre 0 y 200, como resultado de la adición de las variables. Usualmente en este tipo de combinaciones los valores se estandarizan entre 0 y 100 , pero como los porcentajes de cobertura arbórea y los valores de NDVI fueron bajos, y ninguna combinación excedió el valor de 100, no se consideró necesaria la estandarización. 


\section{Análisis estadístico}

Para evaluar el grado de autocorrelación espacial de las variables analizadas, se empleó el índice I de Moran como medida de autocorrelación global, e indicadores locales de asociación espacial (Lisa) como medida de autocorrelación local. La I de Moran, permite analizar el grado de dependencia de los valores que toma una variable en un lugar respecto a los valores de las ubicaciones vecinas. Por su parte, los análisis locales permiten identificar clústeres o agrupaciones de lugares en los que se concentran valores altos de una variable u hotspots, y valores bajos o coldspots (Celemin, 2009). Los análisis geoestadísticos fueron realizados con el software GeoDa ${ }^{\circledR} 1.10$ (Anselin, Syabri y Kho, 2006), definiendo las vecindades de las UPZ por contigüidad y teniendo en cuenta todas las direcciones para su determinación (caso queen). Con el fin de identificar diferencias en el valor del NDVI y el porcentaje de cobertura arbórea de las UPZ según su estrato socioeconómico, y el NDVI de los parques de acuerdo con su clasificación y el estrato socioeconómico donde se localizaron, se aplicó la prueba no paramétrica de Kruskal-Wallis y la prueba post-hoc de Dunn, teniendo en cuenta que los datos no presentaron una distribución normal (Zar, 2010). Estos análisis se realizaron utilizando el programa estadístico R (R Core Team, 2016).

\section{RESULTADOS}

La distribución de la cobertura arbórea en Bogotá evidenció un comportamiento sesgado según el estrato socioeconómico $\left(\mathrm{H}_{(5)}=48.401, p<0.001\right)$. Se identificaron 3 grupos de estratos en los que se observa que las zonas de la ciudad con estratos 1 y 2 presentaron la menor proporción de cobertura arbórea respecto a los demás (figura 3a). El verdor de las UPZ de los diferentes estratos, representado como el NDVI, presentó diferencias significativas $\left(\mathrm{H}_{(5)}=16.5, p<0.005\right)$ pero no se evidenció un patrón ni agrupaciones claras como resultado del análisis post-hoc (figura $3 \mathrm{~b}$ ). Con respecto al estrato de la zona en donde se ubican, el verdor de los parques públicos también presentó diferencias $\left(\mathrm{H}_{(5)}=196.88, p<0.001\right)$, aumentando en los estratos más altos, con excepción del 6 que no fue significativo (figura 3c). El análisis de los parques según su clasificación arrojó diferencias en el verdor $\left(\mathrm{H}_{(3)}=221.15, p<0.001\right)$, presentando una tendencia de disminución en parques más pequeños y con menor área de influencia. Los parques metropolitanos mostraron los valores más altos, seguidos por los zonales y vecinales. Por su parte, los parques de bolsillo, evidenciaron el verdor más bajo (figura $3 d$ ).

El estrato socioeconómico más abundante fue el 3, mientras que el más escaso fue el 6 que se presentó solo en 4 de las 112 UPZ de la ciudad (figura 4a). El porcentaje de cobertura arbórea varió entre 0 y $24 \%$, en donde la mayoría de las UPZ presentaron valores por debajo del $3 \%$ y solamente 4 superaron el $10 \%$ (figura 4b). El NDVI de los parques urbanos presentó una mayor heterogeneidad espacial; sin embargo, se pudo evidenciar al igual que en las anteriores variables una brecha marcada en la que la UPZ con parques urbanos más verdes tuvo un valor más de 6 veces mayor que aquellas con menor abundancia de coberturas vegetales en sus parques (figura 4c). Finalmente, pasando al potencial de regulación de temperatura, presentó un intervalo amplio, con valores que van desde menos de 9 , hasta aproximadamente 84 (figura 4d).

En general, los análisis del índice I de Moran registraron una tendencia débil a la autocorrelación global positiva. En el caso del estrato socioeconómico, se presentó la autocorrelación positiva más alta con un valor de I de Moran de 0.58, la cobertura arbórea registró 0.3 , el potencial de regulación térmico 0.28 y el NDVI de los parques 0.24 . Con un valor de $p<0.001$ para todos los casos, la prueba de hipótesis estadística rechaza la hipótesis nula de aleatoriedad espacial o ausencia de autocorrelación. La figura 5 muestra los resultados de los análisis de LISA univariados para la 

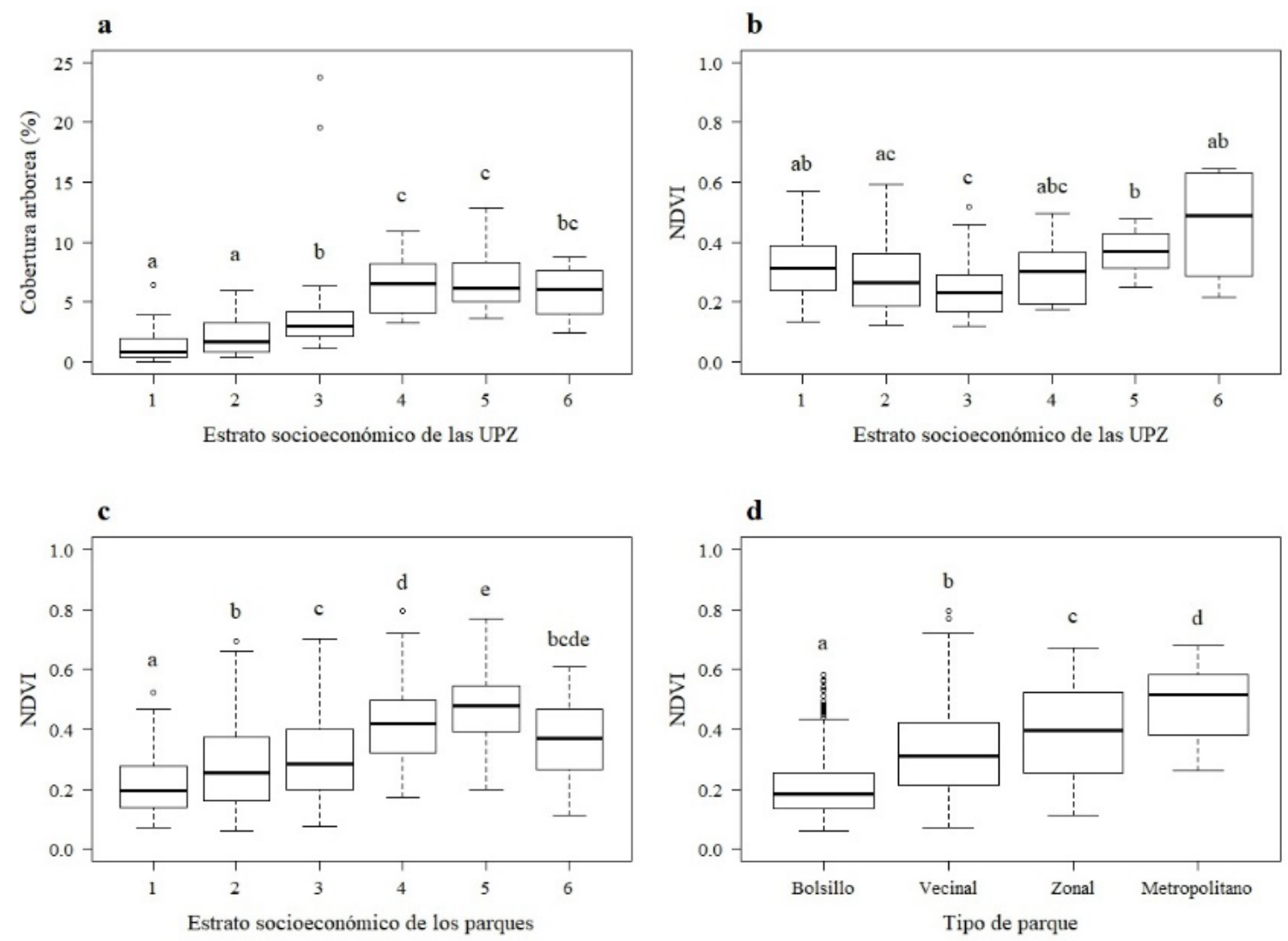

Figura 3. a) Porcentaje de cobertura arbórea en las UPZ de cada estrato socioeconómico; b) NDVI de las UPZ según su estrato socioeconómico; c) NDVI de los parques públicos según el estrato socioeconómico donde se ubican; d) NDVI según tipo de parque urbano. Letras no repetidas representan valores diferentes $(p<0.05)$.
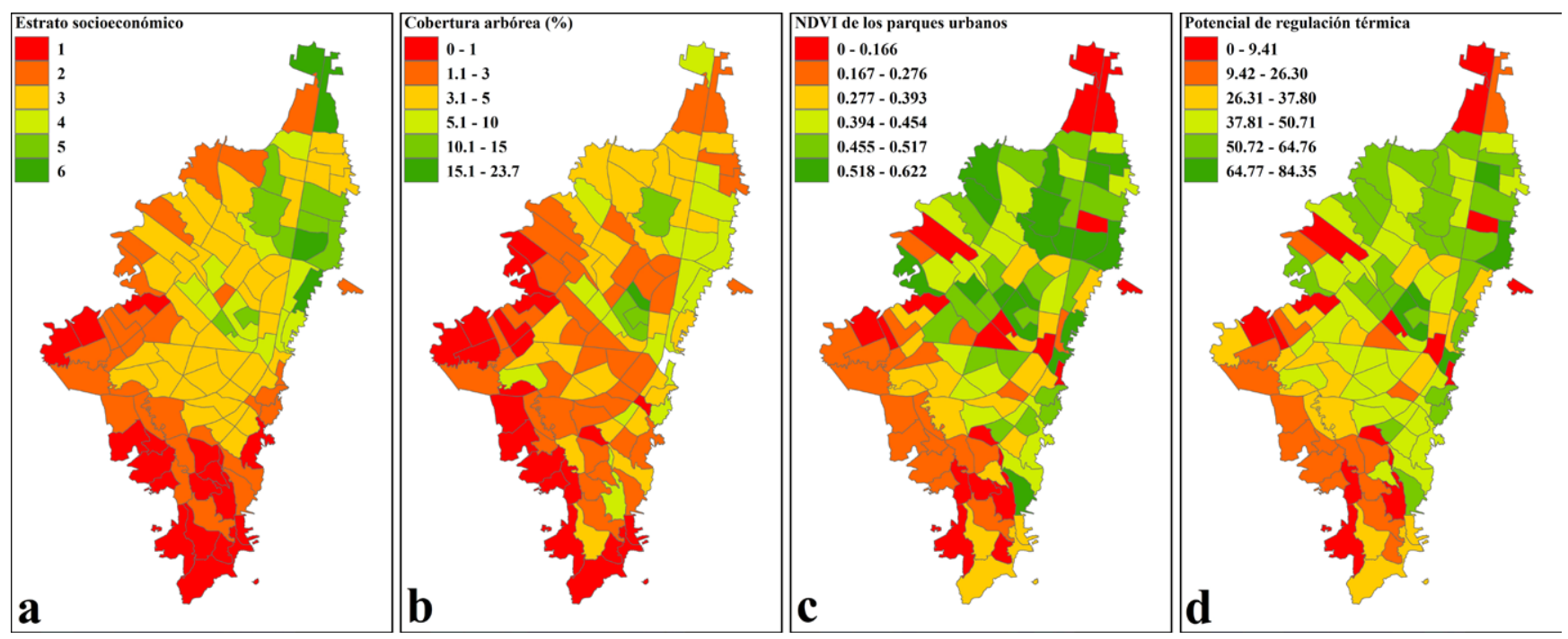

Figura 4. a) Estratificación socioeconómica de la ciudad de Bogotá; b) distribución espacial de la cobertura arbórea de la ciudad; c) media ponderada del NDVI de los parques urbanos; d) puntaje de potencial de regulación térmica. 
determinación de autocorrelación espacial local. Los clústeres del estrato socioeconómico evidencian una clara división de la ciudad, en donde los estratos socioeconómicos altos, se agrupan en la zona norte y los bajos, en la zona sur de la ciudad (figura 5a). La zona suroccidental de la ciudad presentó un coldspot para el porcentaje de cobertura arbórea (figura 5b). Los parques públicos registraron valores bajos de NDVI agrupados principalmente al sur de la ciudad y los valores altos se agruparon predominantemente al norte (figura 5c). Finalmente, el potencial de regulación de temperatura registró nuevamente coldspots al sur de la ciudad y hotspots en la zona norte (figura $5 d$ ). En general, las variables analizadas presentaron intersección de algunas áreas de sus coldspots en el sur y suroccidente, y hotspots en el norte.

\section{DISCUSIÓN}

El presente estudio analizó la distribución de la infraestructura verde pública de la ciudad de Bogotá con respecto a las UPZ, con énfasis en el arbolado y los parques urbanos, y su potencial para regular la temperatura con el fin de evaluar la inequidad ambiental. Aunque otros estudios han analizado la inequidad ambiental de Bogotá (Brown, 2012; Escobedo et al., 2015), ninguno ha analizado la distribución de la cobertura arbórea usando las UPZ como unidad de análisis, ni ha evaluado el verdor con datos obtenidos mediante teledetección. De igual manera, estos análisis han tenido como objetivo evaluar servicios ecosistémicos como la remoción de contaminantes del aire y el almacenamiento de carbono en biomasa, pero ninguno ha relacionado la distribución de las coberturas vegetales urbanas con el potencial de regulación térmica.

Los resultados obtenidos evidencian que la distribución de la infraestructura verde y su potencial para regular la temperatura es heterogénea en la ciudad de Bogotá respecto a los estratos socioeconómicos. En el caso del porcentaje de cobertura arbórea, se evidenció una clara tendencia a la disminución en las áreas con menor estrato socioeconómico. Esto concuerda con estudios realizados previamente en Bogotá, los cuales indicaron que
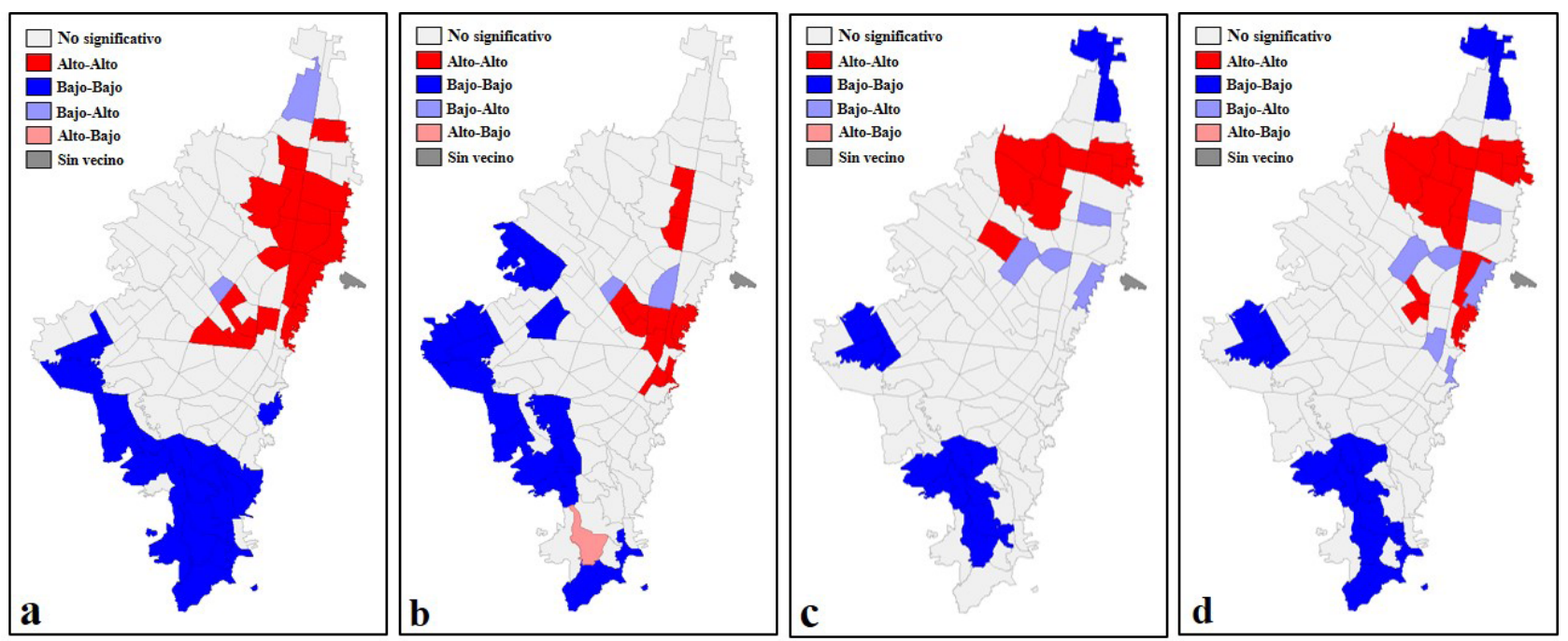

Figura 5. Análisis de LISA univariados (agrupaciones) para las variables analizadas en la ciudad de Bogotá: a) mapa de agrupaciones para el estrato socioeconómico; b) mapa de agrupaciones para el porcentaje de cobertura arbórea pública; c) mapa de agrupaciones para la media ponderada del NDVI de los parques públicos; d) mapa de agrupaciones para el puntaje de potencial de regulación térmica. Resultados significativos con $p<0.05$. 
tanto la cantidad de árboles (Brown, 2012) como su diversidad, área de copa y otros atributos estructurales (Escobedo et al., 2015), son mayores en los estratos más altos. De manera similar, este patrón de mayor abundancia y calidad de la infraestructura verde en las zonas con mejor condición socioeconómica, ha sido observado en otras ciudades de Latinoamérica (De la Maza et al., 2002; Pedlowski et al., 2002; Escobedo et al., 2016), Norteamérica (Schwarz et al., 2015; Nesbitt y Meitner, 2016) y Europa (Kabisch y Haase, 2014). Teniendo en cuenta que los estratos socioeconómicos buscan capturar las diferencias socioeconómicas de la población (Hernández, 2016), los resultados obtenidos indican problemas de inequidad ambiental en Bogotá, tal como han concluido previamente otros autores (Brown, 2012; Escobedo et al., 2015).

Las diferencias en la distribución de la cobertura arbórea se pueden explicar a partir del rápido aumento poblacional que ha experimentado Bogotá durante los últimos años (Cristancho y Triana, 2018) como respuesta a la tendencia global de migración hacia las áreas urbanas (Aide y Grau, 2004), particularmente acentuada en Colombia por decenios de conflicto armado (Shultz et al., 2014). Esto generó un proceso descontrolado de ocupación en las zonas periféricas de la ciudad sin ningún tipo de planificación (Romero, 2010; Muñoz y Ducón, 2016). De acuerdo con Romero (2010), a partir de la década de 1970, el crecimiento de la ciudad por la presión poblacional se direccionó hacía el norte, sur y suroccidente de la ciudad, los dos últimos casos como consecuencia de desplazamientos rurales a zonas urbanas, generando procesos de parcelación, urbanización, edificación desordenada y reducción de áreas libres hasta aproximadamente $5 \%$. Teniendo en cuenta que la historia de los sistemas urbanos, la interacción de sus componentes y la configuración espacial en un momento específico determinan las posibilidades futuras de manejo (Pickett et al., 2017), la ocupación desordenada de las áreas periféricas al sur y suroccidente de la ciudad se refleja actualmente en la baja cantidad de áreas verdes y cobertura arbórea en estas áreas (Brown, 2012; Escobedo et al., 2015).

Se ha reportado que la historia de urbanización en la ciudad de Bogotá no solo afecta la disponibilidad de coberturas vegetales sino también aspectos fundamentales de la flora urbana como la composición y la diversidad de especies en los jardines, y la forma en la que los habitantes de las diferentes áreas interactúan con estos elementos (Sierra-Guerrero y Amarillo-Suárez, 2017). Esta inequidad en el acceso al arbolado urbano implica también inequidad en el disfrute de los múltiples beneficios que esto genera para la salud pública (Ernstson, 2013; Wolch et al., 2014). Mitchell y Popham (2008), además, plantean que los vecindarios con menores ingresos económicos enfrentan una serie de problemáticas asociadas a la salud y mayores tasas de mortalidad, y como consecuencia estas poblaciones vulnerables recibirían un mayor beneficio neto de estas coberturas que aquellas que gozan de mejores capacidades económicas.

Las comparaciones del verdor permiten observar que los estratos 6, 5 y 1 registran los valores más altos respectivamente. Esto puede ser consecuencia de analizar no solo las áreas verdes públicas, sino toda la superficie de las UPZ, teniendo en cuenta que en el caso de los estratos más altos se presenta el mayor número de áreas verdes privadas y en el estrato 1 gran influencia de áreas verdes periféricas de la ciudad. Por otra parte, podría ser el resultado de la heterogeneidad espacial de las ciudades y la resolución espacial de los datos empleados para los análisis (Pickett et al., 2017; Zhou, Pickett y Cadenasso, 2017). Al analizar los parques según su estrato, se observan valores más altos de verdor a medida que incrementa el estrato, con un comportamiento similar a la cobertura arbórea. Nuevamente, esto tiene implicaciones en el disfrute de los beneficios generados por los parques en las diferentes zonas de la ciudad (Tzoulas et al., 2007; Bowler et al., 2010). El NDVI ha sido relacionado inversamente con la temperatura superficial en áreas urbanas (Weng et al., 2004; Weng, 2009; Huang y Ye, 2015) y con toda una 
serie de procesos fisiológicos tales como la capacidad de evapotranspiración, que determinan la funcionalidad de las coberturas vegetales no solo para regular la temperatura sino para la generación de bienestar en las poblaciones humanas (Kerr y Otrovsky, 2003; Glenn et al., 2008; Ayanu et al., 2012). Particularmente en las ciudades, se ha identificado la importancia del NDVI y otras medidas de verdor en los vecindarios, sobre la calidad de vida por su relación con la salud física, psicológica y la sensación de bienestar (Zhou et al., 2009; Leslie et al., 2010). Conjuntamente, lo anterior sugiere que la calidad de estas áreas verdes respecto a su capacidad de regulación de temperatura y a la provisión de otros servicios y beneficios es mayor en las zonas con mayor estrato socioeconómico.

De igual forma, el NDVI cambió de acuerdo con el tipo de parque. Si bien la clasificación de los parques se basa en su tamaño, entre otros criterios (Secretaría Distrital de Planeación, 2009), con este análisis se buscó no solo determinar la calidad de cada tipo de parque para prestar servicios ecosistémicos, sino también la variación del verdor que pueden presentar áreas con el mismo uso del suelo. Los parques metropolitanos presentaron un mayor verdor seguido por los vecinales, los zonales y los de bolsillo; esto significa que, en ese mismo orden, cada categoría tiene un mayor potencial para la oferta de servicios ecosistémicos por unidad de área. Por otra parte, la variación del verdor que existe para esta clase de uso del suelo es una evidencia de las carencias que tienen las estimaciones de servicios ecosistémicos basados en el uso del suelo a escalas pequeñas y en áreas altamente heterogéneas como las ciudades (Eigenbrod et al., 2010), ya que tienen en cuenta la variabilidad existente entre categorías de uso del suelo pero no la variabilidad al interior de cada una de ellas.

Los análisis de autocorrelación espacial indican que la distribución del estrato socioeconómico y las demás variables analizadas no es aleatoria. Esto se confirma con los resultados de los análisis Lisa, que evidencian claramente, primero, la ampliamente reconocida y marcada dualidad socioeconómica entre el norte y el sur de Bogotá (Muñoz y Ducón, 2016); y, segundo, un patrón diferencial en la distribución de la cobertura arbórea, el verdor de los parques y el potencial de regulación térmica. En pocas palabras, los hotspots y coldspots de estas variables se distribuyeron de una forma similar y refuerzan los resultados previamente analizados que indican la presencia de inequidad en la distribución del potencial de regulación de temperatura.

Si bien los resultados aportan conocimiento valioso para comprender la capacidad de regulación de temperatura en las diferentes zonas de la ciudad, la aproximación se basa en el análisis de la oferta. Un análisis más detallado que incluya la identificación de la demanda del servicio en las diferentes zonas de la ciudad en términos de la exposición y la vulnerabilidad a la temperatura, brindaría bases más sólidas para la implementación de acciones más efectivas (Norton et al., 2015). Otro aspecto a tener en cuenta sobre los resultados obtenidos es la fuente de los datos. Se ha identificado que existen diferencias importantes en la cuantificación de la infraestructura verde urbana y los servicios que presta, como consecuencia de las variaciones que presentan las diferentes fuentes de datos y las fallas institucionales en consolidar y manejar datos comunes, entorpeciendo así los procesos de planificación, gestión e investigación (Feltynowski et al., 2018). Bogotá tiene el privilegio de contar con el censo de arbolado urbano, el cual es una herramienta y una fuente de datos de gran valor en la búsqueda de una ciudad más sostenible, por su importancia para el estudio de los servicios ecosistémicos (Nowak et al., 2008; Escobedo et al., 2015). Esto recalca la importancia de contar con este tipo de datos medidos en terreno, lo más actualizados y depurados que sea posible. Aun así, es necesaria investigación adicional que contribuya al entendimiento de la prestación de este y otros servicios ecosistémicos en áreas 
urbanas en lo concerniente a especie, teniendo en cuenta las diferencias en los procesos fisiológicos y los rasgos funcionales, con el fin de determinar las especies más adecuadas para el bienestar del ser humano y la biodiversidad en las ciudades.

\section{CONCLUSIONES}

Se identificaron diferencias en la distribución de la cobertura arbórea y en el verdor de los parques públicos de acuerdo con el estrato socioeconómico de las UPZ. Esto es un indicador de inequidad en la distribución de estos elementos y tiene implicaciones en la provisión del servicio de regulación térmica y otros servicios ecosistémicos en las distintas zonas de la ciudad. Los análisis de autocorrelación espacial global, así como la localización de los hotspots y coldspots de las variables analizadas confirman estas diferencias, dejando en evidencia la marcada y ampliamente reconocida dualidad que existe entre el sur y el norte de la ciudad.

La heterogeneidad en la distribución de la infraestructura verde y el potencial de regulación térmica evidencian la complejidad de los procesos e interacciones que tienen lugar en la ciudad de Bogotá entre los diferentes componentes del ecosistema urbano. Esto constituye un desafío en la búsqueda de una distribución más equitativa de los beneficios que proveen las coberturas vegetales urbanas.

Un factor importante que puede estar motivando la inequidad observada es la historia del desarrollo de las diferentes zonas de la ciudad, que resulta en una configuración actual y determina la gama de posibilidades de manejo y mejora de la infraestructura verde. Lidiar con estas restricciones requiere encontrar soluciones diferentes a las tradicionales y que permitan mejorar el verdor y la calidad de vida de todos los ciudadanos.

Los parques públicos presentaron valores de verdor diferentes de acuerdo con su categoría de clasificación en la estructura ecológica principal. Esto sugiere que la capacidad de proveer servicios ecosistémicos cambia con el tipo de parque. Usualmente, en los estudios de servicios ecosistémicos y en los procesos de manejo y gestión urbana, se consideran las variaciones entre clases de usos del suelo, pero no la variabilidad al interior de estas clases. Considerar las diferencias que existen entre áreas verdes maximizaría los esfuerzos tanto en cuantificar más adecuadamente estos servicios como en garantizar una provisión más equitativa.

Este trabajo aporta información clave para el manejo de la infraestructura verde, la maximización del potencial de regulación térmica y la provisión de otros servicios ecosistémicos, ante escenarios inminentes de cambio climático.

\section{AGRADECIMIENTOS}

El autor agradece al Jardín Botánico de Bogotá por financiar el trabajo y compartir los datos del Sigau. A Ideca y la Secretaria Distrital de Ambiente de Bogotá por poner a disposición del público las capas geográficas empleadas para los análisis. AI Servicio Geológico de los Estados Unidos (USGS) por suministrar la imagen Landsat preprocesada para el desarrollo de este trabajo. A Alexander Bustos y Blanca Caleño por sus valiosos comentarios a las versiones preliminares del manuscrito.

\section{CONFLICTO DE INTERESES}

Los autores declaran no tener conflicto de intereses.

\section{CONTRIBUCIÓN POR AUTOR}

El autor único es responsable de la obra en todos los aspectos que condujeron a la elaboración de su publicación. 


\section{REFERENCIAS}

Aide, T. M. y Grau, H. R. (2004). Globalization, migration and Latin American ecosystems. Science, 305, 1915-1916.

https://doi.org/10.1126/science.1103179

Ángel, L., Ramírez, A. y Domínguez, E. (2010). Isla de calor y cambios espacio-temporales de la temperatura en la ciudad de Bogotá. Revista de la Academia Colombiana de Ciencias Exactas, Físicas y Naturales, 34(131), 173-183.

Anselin, L., Syabri, I. y Kho, Y. (2006). GeoDa: An introduction to spatial data analysis. Geographical Analysis, 38(1), 5-22.

https://doi.org/10.1111/j.0016-7363.2005.00671.x

Ayanu, Y. Z., Conrad, C., Nauss, T., Wegmann, M. y KoeIIner, T. (2012). Quantifying and mapping ecosystem services supplies and demands: A review of remote sensing applications. Environmental Science \& Technology, 46, 8529-8541.

https://doi.org/10.1021/es300157u

Basara, J. B., Basara, H. G., Illston, B. G. y Crawford, K. C. (2010). The impact of the urban heat island during an intense heat wave in Oklahoma City. Advances in Meteorology.

http://doi.org/10.1155/2010/230365

Bolund, P. y Hunhammar, S. (1999). Ecosystem services in urban areas. Ecological Economics, 29, 293-301.

https://doi.org/10.1016/S0921-8009(99)00013-0

Bowler, D. E., Buyung-Ali, L., Knight, T. M. y Pullin, A. S. (2010). Urban greening to cool towns and cities: A systematic review of the empirical evidence. Landscape and urban planning, 97(3), 147-155. https://doi.org/10.1016/j.landurbplan.2010.05.006

Brown, M. (2012). The Bogotá Green Divide: Inequality in Street Tree Coverage Across Estratos. Recuperado de http://www.citinature.org/ uploads/4/7/4/0/4740372/citinature_report_bogota_green_divide.pdf

Burkhard, B., Kroll, F., Müller, F. y Windhorst, W. (2009). Landscapes' capacities to provide ecosystem services - a-concept for Land-Cover based assessments. Landscape online, 15, 1-22.

https://doi.org/10.3097/LO.200915
Burkhard, B., Kroll, F., Nedkov, S. y Müller, F. (2012). Mapping ecosystem service supply, demand and budgets. Ecological Indicators, 21, 17-29.

https://doi.org/10.1016/j.ecolind.2011.06.019

Celemin, J. P. (2009). Autocorrelación espacial e indicadores locales de asociación espacial. Importancia, estructura y aplicación. Revista Universitaria de Geografía, 18(1), 11-31. Recuperado de

http://www.scielo.org.ar/scielo.php?script=sci_arttext\&pid=S1852-42652009000100002

Chander, G., Markham, B. L. y Helder, D. L. (2009). Summary of current radiometric calibration coefficients for Landsat MSS, TM, ETM+, and EO-1 ALI sensors. Remote sensing of environment, 113, 893-903.

https://doi.org/10.1016/j.rse.2009.01.007

Chuvieco, E. (2016). Fundamentals of satellite remote sensing: An environmental approach. CRC press.

Cortinovis, C. y Geneletti, D. (2018). Mapping and assessing ecosystem services to support urban planning: A case study on brownfield regeneration in Trento, Italy. One Ecosystem, 3, e25477.

https://doi.org/10.3897/oneeco.3.e25477

Cristancho, C. y Triana, E. (2018). Análisis demográfico y proyecciones poblacionales de Bogotá. Bogotá: Secretaría Distrital de Planeación. Recuperado de http://www.sdp.gov.co/sites/default/files/demografia_proyecciones_2017_0_0.pdf

Dawes, L. C., Adams, A. E., Escobedo, F. J. y Soto J. R. (2018). Socioeconomic and ecological perceptions and barriers to urban tree distribution and reforestation programs. Urban Ecosystems, 21(4), 657-671.

https://doi.org/10.1007/s11252-018-0760-z

De la Maza, C. L., Hernández, J., Brown, H., Rodríguez, M. y Escobedo, F. (2002). Vegetation diversity in the Santiago de Chile urban ecosystem. Arboricultural Journal, 126(4), 347-357.

https://doi.org/10.1080/03071375.2002.9747349

Dirpen (2006). Manual del censista y auxiliar: censo del árbol urbano de Bogotá D.C. Bogotá: Jardín Botánico de Bogotá José Celestino Mutis, Departamento Nacional de Estadística.

Egoh, B., Drakou, E. G., Dunbar, M. B., Maes, J. y Willemen, L. (2012). Indicators for Mapping Ecosystem 
Services: A Review. Luxemburgo: European Comission, Joint Research Centre.

Egoh, B., Reyers, B., Rouget, M., Richardson, D. M., Le Maitre, D. C. y van Jaarsveld, A. S. (2008). Mapping ecosystem services for planning and management. Agriculture, Ecosystems and Environment, $127,135-140$

https://doi.org/10.1016/j.agee.2008.03.013

Eigenbrod, F., Armsworth, P. R., Anderson, B. J., Heinemeyer, A., Gillings, S., Roy, D.V., ... y Gaston, K. J. (2010). The impact of proxy-based methods on mapping the distribution of ecosystem services. Journal of Applied Ecology, 47, 377-385.

https://doi.org/10.1111/j.1365-2664.2010.01777.x

Ellis, E. C. y Ramankutty, N. (2008). Putting people in the map: anthropogenic biomes of the world. Frontiers in Ecology and the Environment, 6(8), 439-447. https://doi.org/10.1890/070062

Ernstson, H. (2013). The social production of ecosystem services: A framework for studying environmental justice and ecological complexity in urbanized landscapes. Landscape and Urban Planning, 109(1), 7-17.

https://doi.org/10.1016/j.landurbplan.2012.10.005

Escobedo, F. J., Clerici, N., Staudhammer, C. L. y Corzo, G. T. (2015). Socio-ecological dynamics and inequality in Bogotá, Colombia's public urban forests and their ecosystem services. Urban Forestry and Urban Greening, 14, 1040-1053.

https://doi.org/10.1016/j.ufug.2015.09.011

Escobedo, F. J., Palmas-Perez, S., Dobbs, C., Gezan, S. y Hernández, J. (2016). Spatio-Temporal Changes in Structure for a Mediterranean Urban Forest: Santiago, Chile 2002 to 2014. Forests, 7, 121.

https://doi.org/10.3390/f7060121

Feltynowski, M., Kronenberg, J., Bergier, T., Kabisch, N., Łaszkiewicz, E. y Strohbach, M. W. (2018). Challenges of urban green space management in the face of using inadequate data. Urban Forestry and Urban Greening, 31, 56-66.

https://doi.org/10.1016/j.ufug.2017.12.003

Foley, A., DeFries, R., Asner, G., Barford, C., Bonan, G., Carpenter, S., ... y Snyder, P. (2005). Global consequences of land use. Science, 309, 570-574.

https://doi.org/10.1126/science.1111772
Glenn, E. P., Huete, A. R., Nagler, P. L. y Nelson, S. G. (2008). Relationship between remotely-sensed vegetation indices, canopy attributes and plant physiological processes: What vegetation indices can and cannot tell us about the landscape. Sensors, 8, 2136-2160.

https://doi.org/10.3390/s8042136

Gómez-Baggethun, E. y Barton, D.N. (2013). Classifying and valuing ecosystem services for urban planning. Ecological Economics, 86, 235-245.

https://doi.org/10.1016/j.ecolecon.2012.08.019

Gómez-Baggethun, E., Gren, Å., Barton, D. N., Langemeyer, J., McPhearson, T., O'Farrell, P., ... y Kremer, P. (2013). Urban ecosystem services. En T. Elmqvist, M. Fragkias, J. Goodness, B. Güneralp, P.J. Marcotullio, R.I. McDonald, ... C. Wikinson (eds.), Urbanization, Biodiversity and Ecosystem Services: Challenges and Opportunities (pp. 175251). Dordrecht: Springer.

Grimmond, S. U. E. (2007). Urbanization and global environmental change: local effects of urban warming. The Geographical Journal, 173(1), 83-88.

https://doi.org/10.1111/j.1475-4959.2007.232_3.x

Hardin, P. J y Jensen, R. R. (2007). The effect of urban leaf area on summertime urban surface kinetic temperatures: a Terre Haute case study. Urban Forest Urban Green, 6, 63-72.

https://doi.org/10.1016/j.ufug.2007.01.005

Hernández, I. (ed.) (2016). La estratificación en Bogotá: impacto social y alternativas para asignar subsidios. Bogotá: Secretaría Distrital de Planeación.

Huang, C. y Ye, X. (2015). Spatial Modeling of urban vegetation and land surface temperature: A case study of Beijing. Sustainability, 7, 9478-9504.

https://doi.org/10.3390/su7079478

Isaacs, P. J. y Jaimes, V. I. (2014). Análisis multitemporal de la estructura del paisaje del Distrito Capital, años 1991-2012. Bogotá: Jardín Botánico de Bogotá José Celestino Mutis.

Jensen, J. R. (2000). Remote sensing of the environment: An earth resource perspective. Upper River: Prantice Hall.

Jim, C. Y. y Chen, W. Y. (2009). Ecosystem services and valuation of urban forests in China. Cities, 26, 187-194.

https://doi.org/10.1016/j.cities.2009.03.003 
Kabisch, N. y Haase, D. (2014). Green justice or just green? Provision of urban green spaces in Berlin, Germany. Landscape and Urban Planning, 122, 129-139.

https://doi.org/10.1016/j.landurbplan.2013.11.016

Kerr, J. T. y Ostrovsky, M. (2003). From space to species: Ecological applications for remote sensing. Trends in Ecology and Evolution, 18(6), 299-305.

https://doi.org/10.1016/S0169-5347(03)00071-5

Leslie, E., Sugiyama, T., lerodiaconou, D. y Kremer, P. (2010). Perceived and objectively measured greenness of neighbourhoods: Are they measuring the same thing? Landscape and Urban Planning, 95, 28-33.

https://doi.org/10.1016/j.landurbplan.2009.11.002

Livesley, S. J., McPherson, E. G. y Calfapietra, C. (2016). The urban forest and ecosystem services: Impacts on urban water, heat, and pollution cycles at the tree, street, and city scale. Journal of Environmental Quality, 45(1), 119-124.

https://doi.org/10.2134/jeq2015.11.0567

Lovell, S. T. y Taylor, J. R. (2013). Supplying urban ecosystem services through multifunctional green infrastructure in the United States. Landscape Ecology, 28, 1447-1463.

https://doi.org/10.1007/s10980-013-9912-y

Maes, J., Egoh, B., Willemen, L., Liquete, C., Vihervaara, P., Schägner, J.P., ... y Bidoglio, G. (2012). Mapping ecosystem services for policy support and decision making in the European Union. Ecosystem Services, 1, 31-39.

\section{https://doi.org/10.1016/j.ecoser.2012.06.004}

Maes, J., Polce, C., Zulian, G., Vandecasteele, I., Perpiña, C., Rivero, I.M., Guerra, C., Vallecillo, S., Vizcaino, P. y Hiederer, R. (2017). Mapping Regulating Ecosystem Services. En J. Burkhard y J. Maes (eds.), Mapping Ecosystem Services (pp. 179-188). Sofia: Pensoft Publishers.

Mahecha, G., Sánchez, F., Chaparro, J., Cadena, H., Tovar, G., Villota, L., Morales, G., Castro, J., Bocanegra, F. y Quintero, M. (2010). Arbolado urbano de Bogotá: Identificación, descripción y bases para su manejo. Bogotá: Secretaría Distrital de Ambiente.
McPhearson, T. (2011). Toward a sustainable New York City: Greening through urban forest restoration. En E. Slavin (ed.), Sustainability in America's Cities: Creating the Green Metropolis (pp. 181-204). Washington: Island Press.

Mitchell, R. y Popham, F. (2008). Effect of exposure to natural environment on health inequalities: An observational population study. Lancet, 372, 1655-1660.

https://doi.org/10.1016/S0140-6736(08)61689-X

Muñoz, J. H. y Ducón, J. C. (2016). Análisis econométrico espacial de las localidades de Bogotá y municipios del borde urbano. Criterios, 9(2), 129-157. Recuperado de

https://doi.org/10.21500/20115733.3088

Nesbitt, L. y Meitner, M. J. (2016). Exploring relationships between socioeconomic background and urban greenery in Portland, OR. Forests, 7, 162.

https://doi.org/10.3390/f7080162

Norton, B. A., Coutts, A. M., Livesley, S. J., Harris, R. J., Hunter, A. M. y Williams, N. (2015). Planning for Cooler cities: A framework to prioritise green infrastructure to mitigate high temperatures in urban landscapes. Landscape and Urban Planning, 134, 127-138.

https://doi.org/10.1016/j.landurbplan.2014.10.018

Nowak, D. J., Crane, D. E., Stevens, J. C., Hoehn, R. E., Walton, J. T. y Bond, J. (2008). A ground based method of assessing urban forest structure and ecosystem services. Arboriculture \& Urban Forestry, 34(6), 347-358.

Ochoa, V. y Urbina-Cardona, N. (2017). Tools for spatially modeling ecosystem services: Publications trends, conceptual reflections and future challenges. Ecosystem Services, 26, 155-169.

https://doi.org/10.1016/j.ecoser.2017.06.011

Patz, J. A., Campbell-Lendrum, D., Holloway, T. y Foley, J. A. (2005). Impact of regional climate change on human health. Nature, 438, 310-317.

https://doi.org/10.1038/nature04188

Pedlowski, M. A., Da Silva, V. A., Adell, J. J. y Heynen, N. C. (2002). Urban forests and environmental inequality in Campos Dos Goytacazes, Rio de Janeiro, Brazil. Urban Ecosystems, 6, 9-20.

https://doi.org/10.1023/A:1025910528583 
Peng, S., Piao, S., Clais, P., Friedlingstein, P., Ottle, C., Bréon, F. M., ... y Myneni, R. B. (2012). Surface Urban Heat Island Across 419 Global Big Cities. Environmental Science and Technology, 46, 696-703. https://doi.org/10.1021/es2030438

Pickett, S. T. A., Cadenasso, M. L., Rosi-Marshall, E. J., Belt, K. T., Groffman, P. M., Grove, J. M., ... y Swan, C. M. (2017). Dynamic heterogeneity: a framework to promote ecological integration and hypothesis generation in urban systems. Urban Ecosystems, 20(1), 1-14.

https://doi.org/10.1007/s11252-016-0574-9

QGIS Development Team (2011). QGIS Geographic Information System. Beaverton: Open Source Geospatial Foundation Project. Recuperado de

http://qgis.osgeo.org

R Core Team (2016). R: A language and environment for statistical computing. Viena: R Foundation for Statistical Computing. Recuperado de

https://www.R-project.org/

Rizwan, A. M., Dennis, Y. C. L. y Liu, C. (2008). A review on the generation, determination and mitigation of urban heat islands. Journal of Environmental Sciences, 20, 120-128.

https://doi.org/10.1016/S1001-0742(08)60019-4

Rodríguez-Laguna, R., Meza-Rangel, J., Vargas-Hernández, J. y Jiménez-Pérez, J. (2009). Variación en la cobertura de suelo en un ensayo de procedencias de Pinus greggii Engelm. en el cerro El Potosí, Galeana, Nuevo León. Madera y Bosques, 15(1), 47-59. Recuperado de

http://www.scielo.org.mx/scielo.php?pid= S1405-047120 09000100004\&script=sci_abstract\&tlng=en

Romero, J. A. (2010). Transformación urbana de la ciudad de Bogotá, 1990-2010: efecto espacial de la liberalización del comercio. Perspectiva Geográfica, 15, 85-112.

Rouse, J. W., Haas, R. W., Schell, J. A., Deering, D. W. y Harlan, J. C. (1974). Monitoring the vernal advancement and retrogradation (Greenwave effect) of natural vegetation. Greenbelt: NASA/GSFCT.

Santamouris, M., Cartalis, C., Synnefa, A. y Kolokotsa, D. (2015). On the impact of urban heat island and global warming on the power demand and electricity consumption of buildings - A review. Energy and Buildings, 98, 119-124.

https://doi.org/10.1016/j.enbuild.2014.09.052

Schneiders, A., Van Daele, T., Van Laduyt, W. y Van Reeth, W. (2012). Biodiversity and ecosystem services: Complementary approaches for ecosystem management? Ecological Indicators, 21, 123-133.

https://doi.org/10.1016/j.ecolind.2011.06.021

Schwarz, K., Fragkias, M., Boone, C. G., Zhou, W., McHale, M., Grove, J. M., ... y Ogden, L. (2015). Trees grow on money: urban tree canopy cover and environmental justice. PLoS One, 10(4).

https://doi.org/10.1371/journal.pone.0122051

Secretaría Distrital de Planeación (2009). Conociendo Bogotá y sus localidades: resumen de los principales aspectos físicos, demográficos y socioeconómicos. Bogotá: Alcaldía Mayor de Bogotá. Recuperado de http://oab.ambientebogota.gov.co/es/con-la-comunidad/ ES/cartilla-conociendo-las-localidades-de-bogota

Shashua-Bar, L. y Hoffman, M. E. (2000). Vegetation as a climatic component in the design of an urban street: An empirical model for predicting the cooling effect of urban green areas with trees. Energy and Buildings, 31(3), 221-235

Shultz, J. M., Garfin, D. R., Espinel, Z., Araya, R., Oquendo, M. A., Wainberg, M. L., ... y Wilson, F. E. (2014). Internally displaced "victims of armed conflict" in Colombia: the trajectory and trauma signature of forced migration. Current Psychiatry Reports, 16(10), 475.

Sierra-Guerrero, M. C. y Amarillo-Suárez, A. R. (2017). Socioecological features of plant diversity in domestic gardens in the city of Bogotá, Colombia. Urban Forestry and Urban Greening, 28, 54-62.

https://doi.org/10.1016/j.ufug.2017.09.015

Storey, J., Choate, M. y Lee, K. (2014). Landsat 8 Operational Land Imager On-Orbit Geometric Calibration and Performance. Remote Sensing, 6, 11127-11152.

https://doi.org/10.3390/rs61111127

Tzoulas, K., Korpela, K., Venn, S., Yli-Pelkonen, V., Kaźmierczak, A., Niemela, J. y James, P. (2007). 
Promoting ecosystem and human health in urban areas using Green Infrastructure: A literature review. Landscape and Urban Planning, 81(3), 167-178.

https://doi.org/10.1016/j.landurbplan.2007.02.001

United Nations (2018). World Urbanization Prospects: The 2018 Revision. Nueva York: Department of Economic and Social Affairs, Population Division.

United States Geological Survey (2017). Product guide: Landsat 8 Surface Reflectance Code (LaRSC) Product. Sioux Falls: Department of Interior.

Weng, Q. (2009). Thermal infrared remote sensing for urban climate and environmental studies: Methods, applications and trends. ISPRS Journal of Photogrammetry and Remote Sensing, 64(4), 335-344.

https://doi.org/10.1016/j.isprsjprs.2009.03.007

Weng, Q., Lu, D. y Schubring, J. (2004). Estimation of land surface temperature - vegetation abundance relationship for urban heat island studies. Remote Sensing of Environment, 89(4), 467-483.

https://doi.org/10.1016/j.rse.2003.11.005

Wilson, N. R., Norman, L. M., Villareal, M., Gass, L., Tiller, R. y Salywon, A. (2016). Comparison of remote sensing indices for monitoring of desert cienegas. Arid Land Research and Management, 30(4), 460-478.

https://doi.org/10.1080/15324982.2016.1170076

Wolch, J. R., Byrne, J. y Newell, J. P. (2014). Urban green space, public health, and environmental justice: The challenge of making cities 'just green enough'. Landscape and Urban Planning, 125, 234-244. https://doi.org/10.1016/j.landurbplan.2014.01.017

Zar, J. H. (2010). Biostatistical analysis. Upper Saddle River: Prentice Hall.

Zardo, L., Geneletti, D., Pérez-Soba, M. y Van Eupen, M. (2017). Estimating the cooling capacity of Green infrastructures to support urban planning. Ecosystem Services, 26, 225-235.

https://doi.org/10.1016/j.ecoser.2017.06.016

Zhao, S., Da, L., Tang, Z., Fang, H., Song, K. y Fang, J. (2006). Ecological consequences of rapid urban expansion: Shanghai, China. Frontiers in Ecology and the Environment, 4(7), 341-346.

https://doi.org/10.1890/1540-9295(2006)004[0341:ECORUE]2.0.CO;2

Zhou, W., Pickett, S. T. y Cadenasso, M. L. (2017). Shifting concepts of urban spatial heterogeneity and their implications for sustainability. Landscape ecology, 32(1), 15-30.

https://doi.org/10.1007/s10980-016-0432-4

Zhou, W., Troy, A., Grove, J. M. y Jenkins, J. C. (2009). Can money buy green? Demographic and socioeconomic predictors of lawn-care expenditures and lawn greenness in urban residential areas. Society and Natural Resources, 22(8), 744-760.

https://doi.org/10.1080/08941920802074330

Zulian, G., Liekens, I., Broekx, S., Kabisch, N., Kopperoinen, L. y Geneletti, D. (2017). Mapping urban ecosystem services. En J. Burkhard y J. Maes (eds.), Mapping Ecosystem Services (pp. 312-318). Sofia: Pensoft Publishers.

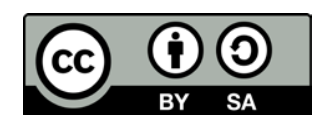

